\title{
Methodology and the Professional Doctorate - the muddy waters of knowledge creation, transfer and workplace capital.
}

The professional doctorate creates opportunities for shared research and novel findings to inform and shape the workplace and cultivate professional capital. In this paper I discuss my own journey in undertaking an Educational doctorate. I critique how my methodological approach enabled me to successfully cross the range of boundaries that I encountered along my way. As I crossed the boundaries between scientist, teacher and researcher, my chosen approach afforded a framework that appealed to my embedded 'positivist tendencies'. It permitted me to acknowledge the varied positioning of both myself and others, and the professional learning that takes place within and across each boundary. I was able to question what constitutes knowledge and what the most acceptable approaches to knowledge creation are when boundaries and research paradigms are crossed. This journey provided me with a greater understanding of how and why as individuals we position ourselves in our roles. This understanding underpins my recommendations for supporting professional learning. I discuss the relevance and potential impact of my findings for both practice and for those undertaking an educational doctorate.

Keywords: Constructivist grounded theory, practitioner training, boundary crossing, professional learning, professional doctorate

\section{Introduction}

A wide range of academic fields now offer professional doctorates as an alternative to the more traditional $\mathrm{PhD}$ route. This route enables practitioners to research their own practice, developing a critical understanding of professional knowledge and professional roles within the wider knowledge market and economy (Usher, 2002). In 
undertaking the doctoral journey, the practitioner is likely to experience tensions between their professional and research roles and changes within their own positioning within these roles. There is a need for constant reflexive self-questioning and this in turn often reveals personal, professional, cultural and methodological divides (Burnard et al 2018, p41). An epistemological challenge is created when an individual's research spans a diversity of professional domains and multi-professional spaces. What constitutes knowledge and what are the most acceptable approaches to knowledge creation when boundaries and research paradigms are crossed?

In this paper I provide a reflective account of my own doctoral research journey and how adopting an appropriate methodological approach supported me in negotiating the boundaries between the professional domains and spaces in which I practised and researched. I document how I evolved as an educational researcher and developed an interpretative portrayal and deeper understanding of the implementation of preregistration training in my field of research. I use the term evolve since it implies a gradual process of growth and change and suggests advancement or improvement. I chart this evolutionary journey from my initial positioning as a biomedical science practitioner in a working environment strongly influenced by positivism to one where I developed an appreciation of the role of socio-cultural interactions and subjectivity in and for developing professional learning and practice. I acknowledge the barriers and opportunities that I encountered along the way and how this led to a more nuanced appreciation of learning and teaching for professional practice.

\section{Context of my research}

My research explored the delivery of a BSc Applied Biomedical Science award at a post-1992 University and linked to pre-registration training for entry onto the professional register for biomedical scientists. My aim was to provide an insight into 
how the programme's stakeholders (academics, biomedical science practitioners and students) interact with the pressures of both internal and external influences and the impact this has upon behaviours and strategies adopted to support learning and teaching on the award. At the heart of my research was a drive to understand how this positioning of stakeholders influences programme delivery, and ultimately the development of capable biomedical science practitioners (Author, 2018).

In undertaking the doctoral journey I moved from scientist to 'a scientist who does educational research too!' The emphasis that I place upon being someone with more than one 'hat' will become clear as I identify how my choice of methodology supported this journey and the relevance and potential impact of my experiences and findings for practice in both my field and others where individuals are undertaking a professional doctorate, negotiating a range of boundaries.

\section{My journey}

As a registered biomedical scientist, I had practiced in the NHS for over 16 years in both a technical and teacher/trainer role before I moved into the higher education sector as a senior lecturer just over 12 years ago. After three years in this post I also became a student undertaking an Educational Doctorate. As outlined above, my research focused upon exploring how key stakeholders on a programme leading to professional registration position themselves in their role and the subsequent impact this has upon teaching and learning for professional practice. Positioning in this context is used to embrace and define an individual's perception of roles and responsibilities and to articulate the approaches individuals themselves adopt as they take on these various roles. Positioning, therefore, affects approaches taken to support 'knowing' and 'doing' for practice as well as how we make sense of our learning experiences. I did not realise as I started on my professional doctorate how my own positioning and coming to 
appreciate 'how' and 'why' would be a major part of this journey. Self-realisation and my own positioning proved to be an initial hurdle. I found myself wearing a range of 'different hats' (practitioner, academic, student and researcher) resulting in tensions and issues of identity. The main barrier that I grappled with in undertaking a professional doctorate was not related to time or the pressures of having a range of roles which were the main worries I had when I first 'signed up'. I found that my main challenge was having to reconcile my view of knowledge and how I came to that knowledge with the views and accepted practices in my new roles. These were not emotions or challenges that I had anticipated or that had been highlighted by my supervisory team. I was crossing multiple boundaries. Wearing my 'day job hat' I was a scientist positioned firmly within an evidence-based culture. This culture also reflected the epistemological and ontological lens through which I had come to view my practitioner role. As an educational researcher I found myself needing to learn a completely new language as well as reconciling my background in evidence-based practice with an interpretative approach. With my scientist hat on I viewed knowledge as something gained through observable and measurable facts. As an educational researcher I was expected to adopt an approach that acknowledged and embraced subjectivity and personal experience. In crossing professional boundaries and transitioning from the periphery to core membership (Wenger, 1998) of this new community, which appeared to sit firmly in a different paradigm, I found that I needed to address my self-understanding and look deeply at my underlying assumptions.

Shifting positions and crossing boundaries, building relationships of trust underlines professional learning but we often overlook the challenges and opportunities afforded as we make these crossings. In the context of professional learning a boundary can be defined as a sociocultural difference, a place where roles or perspectives differ 
between two sites in a way that challenges individuals. However, at the same time there is some sameness (Akkerman and Bakker, 2011). I was certainly feeling challenged as I began my professional doctorate, and at first, I felt unable to draw upon my previous experiences and knowledge. I acknowledged a feeling of suddenly becoming a novice after years of being a confident practitioner in my field of practice. Like many others undertaking a professional doctorate, this was a difficult position to find myself in and I struggled to find a 'sameness' between my previous and new communities of practice in order to be able to make this transition to educational researcher and to gain core membership of this community (Wegner, 1998). I faced a range of challenges. Could my past experiences, knowledge and skills support me on my new journey? Would I be able to draw upon and embed them into my practice as I transitioned into this unfamiliar field? I did not want to have to leave all this experience behind me as I faced my new challenge.

\section{Choosing my methodology}

My research focus required me to adopt a methodological approach that would enable me to draw out both meaning and understanding of complex human experiences in the workplace whilst also addressing the influence of organisational structures and relationships on the construction of communities of practice and learning environments. Like all good students I reviewed the work of others within this field. I found that phenomenology, discourse analysis, and constructivist grounded theory (CGT) had all been widely drawn upon within the healthcare setting when studying social situations and the experiences of individuals (Starks and Trinidad, 2007). They each start with distinct instances of human experiences and slowly unpick these. Discourse analysis focuses upon the use of language and how individuals use language to accomplish their goals within the studied situation. Phenomenological analysis seeks to provide a 
descriptive understanding and 'true to life' conceptualisation of the experience (Holstein and Gubrium, 2005, p.485). In contrast, CGT develops explanatory theories of the studied situation. It assumes that meaning must be constructed, and the researcher moves from initial descriptive analysis to higher level abstractions. This is supported by the development of theoretical categories that allow explanatory models to be constructed (Charmaz, 2005, p.509). It takes a 'reflexive stance on modes of knowing and representing studied life' and does not assume that data 'simply await discovery in an external world' (Charmaz, 2005, p.509). A grounded theory approach allows the researcher to see past the empirical process and develop a deeper understanding and so a picture of 'the whole'. Therefore, unlike phenomenology and discourse analysis, it enables the researcher to move beyond the experiences of the individual practitioners to develop a deeper understanding of the multiple interactions that occur during their dayto-day practice.

CGT resonated more with me as an appropriate methodological approach to enable me to draw out both meaning and understanding of the experiences in the workplace and allowing me to address the external influences on practice and the construction of learning environments. However, choosing a research methodology is often described as being one of the most difficult and confusing decisions that a researcher makes (Opoku, Ahmed and Akotia, 2016, p.32). The literature emphasises the importance of selecting an approach and methods that reflect both yourself as a researcher and your role in the world that you are researching. My choice of methodology not only needed to be based upon my own convictions, beliefs and interests (Goulding, 2002), reflecting my epistemological concerns and norms of practice - but more important to me as an individual, it needed to support me on my research journey and not be a barrier for me as I negotiated qualitative approaches. 
Essentially, I did not want to feel like a complete novice. The whole idea of a professional doctorate is to shape the workplace and cultivate professional capital. I needed an approach that would allow me to draw upon my prior knowledge and experience as I navigated my way through the muddy waters of the professional doctorate and to develop as an educational researcher. In adopting a CGT approach, I felt empowered as a researcher to recognise my previous experiences and perspectives and acknowledge the potential impact that these have on both data gathering and development of theory (Bryant and Charmaz, 2012, p.51). In addition, I was afforded an appreciation of my previous experiences rather than be required to put them to one side. I was able to bring my experiences and knowledge to my research. This is an essential consideration for those starting out on their research journey. As experienced practitioners we have a wealth of skills and knowledge and have developed competence in our role. The position of conscious incompetence as a doctoral student is quite daunting; not having to abandon familiar tools and methods supports the individual as they cross practice boundaries.

As I started out on my journey I brought with me part of my previous world enabling me to build confidence in my new world. I grew as I progressed on my research journey and as I explain below, my prior experiences had a central role within this journey.

\section{Is it what you do or how you do it?}

As I started my research employing familiar methods allowed me to develop an innovative approach to data gathering. Charmaz states that:

Similar to a camera with many lenses, first you view a broad sweep of the landscape. Subsequently, you change your lens several times and shorten your 
focal points to bring key scenes closer and closer into view (Charmaz, 2014, p26)

She stresses that it is not so much about the methods that you adopt for your research the important point is 'how researchers use methods' (Charmaz, 2014, p.2). I drew upon this advice to select a novel approach to my data gathering, drawing upon my background as a scientist.

\section{Using familiar methods and tools}

The ability to draw upon different methods for data collection was very important to me. My research participants, like me, were scientists and more familiar with evidence-based approaches. I was aware that many colleagues would challenge results that were not quantitative and statistically significant; reproducibility and validity being important concepts within their field of work. I took an initial decision to start my research journey by gathering quantitative data via a questionnaire. My motives behind this were two fold. Firstly I did not wish to alienate my research participants through introducing qualitative approaches. Secondly, I personally needed to feel selfassured in both the approach that I was adopting and in interpreting the data I would be gathering. I had used questionnaires before, so they were familiar to me. I added some questions with free text responses which allowed me to also gather some initial qualitative data for analysis. If I am being honest, the gathering of quantitative data provided me with a sense of security - maybe a fall-back position if I failed at qualitative analysis? I was gently dipping my toe into the muddy waters of qualitative analysis and not diving straight in and potentially drowning. Just as important as providing me with a fall-back position, the gathering of quantitative data enabled me to present data graphically as an 'ice-breaker tool' for stimulating discussion in the focus group sessions with my research participants. I provided them, and myself, with an 
object that we recognised and were confident in reviewing - a 'boundary object' that formed a bridge along which I could guide myself, and my participants, to stimulate a more interpretative approach to the research.

\section{Boundary objects}

The notion of 'boundary object' was originally developed to explain collaborations within scientific communities (Star and Griesemer, 1989). It can best be defined as something that has the capacity to be understood by individuals in more than one setting, able to be adapted to local needs and the constraints of the different groups using them, yet still to maintain their identify across the sites (Star and Griesemer, 1989 p.393). I embraced a 'boundary-object' to support my own transition as well as enabling the stakeholders to feel able to participate and collaborate. I found that my initial presentation of quantitative data as an 'ice-breaker tool' acted as a suitable boundary object that resonated with the scientists in the focus groups and encouraged their participation in these sessions. Essentially, the familiarity of quantitative representations (e.g. graphs and pie charts) stimulated greater discussion enabling me to gather individuals' perceptions and gather the more nuanced meanings behind the quantitative data. I soon found my confidence building in the focus groups: we all felt at home with and 'understood' graphical data. As I settled into this new role, I developed my ability to move away from the objective stance of the numbers gathered to question 'why', 'what' and 'how' to gain a more subjective interpretation of individuals positioning.

From a personal perspective, my initial approach of using familiar methods also provided what could best be described as a 'comfort blanket' as I attempted to straddle and negotiate positivist and interpretivist paradigms.

Data Analysis - one step at a time. 
Unlike research approaches I had adopted before, where data gathering is completed before analysis is undertaken, a CGT approach requires constant data gathering and analysis of data. This is where my choice of methodology really afforded me a lifeline. Not only did it allow me to take with me on my journey some familiar methods and tools which facilitated confidence building, supporting my transition between what at first appeared to me as two conflicting paradigms. It also did not require me to master qualitative analysis from the start. I was able to develop this new skill without it impacting upon the trustworthiness of data interpretation. In a CGT approach data is gathered and analysed as you progress. Constant comparative methods are used to establish analytic differences and so allow comparisons to be made at every level of analytic work (Charmaz, 2014, p.132). Codes are created to describe the data gathered and these are used to create categories. Codes are compared to codes, codes to categories and categories to categories allowing an inductive approach. Abductive reasoning also occurs at all stages of analysis and is an essential feature of constant comparative analysis to support theoretical integration (Birks and Mills, 2011, p11). As I started to analyse the data I was gathering I found that I was again able to draw upon rather than reject the skills that I had developed as a scientist. My scientific background aligned naturally to an approach that used flow-charts and diagramming to link data from a range of sources, assisting me to make conceptual links and compare stakeholders' perceptions of situations and roles. A major learning point came as I became aware that as we cross boundaries we do not need to lose our unique personal or professional identity but to develop ways of embedding them into our newly developing professional identity; building on the skills that we already have and using these to develop new knowledge and understanding. I drew upon my strengths as a scientist to support me to cross the boundary into educational researcher. It is essential for those 
embarking upon professional doctorates to acknowledge positioning to allow greater critical understanding of professional roles within the wider picture.

As a scientist I was familiar with maintaining a laboratory logbook to record my activities, findings and data for analysis. I soon found that this is no different when adopting a qualitative approach to research - it is just in a different guise! Holton (2012, p.281) discusses the value of continual memo writing as a tool that 'helps to raise the data to a conceptual level and develop the properties of each category'. Maintaining a continual memo helps in guiding the next steps in further data gathering, coding and analysis. As I started to develop as an educational researcher my 'scientific logbook' evolved into detailed memos and an invaluable reflective journal. This journal was vital in supporting me on my research journey and its evolution from simple notes and jottings highlighting possible ideas or areas for further investigation to in-depth reflexive conversations with myself was instrumental in supporting me to develop theoretical insights into the empirical data. Memo writing assisted in developing existing categories, examining codes and an understanding of the relationships between the categories I was creating. Clarke (2005, p.85) describes them as intellectual capital in the bank'. They form an intrinsic feature of the iterative approach of CGT encouraging the researcher to stand back and focus on the codes, dissecting and comparing them and allowing links to be formed. For me it was a way of standing back from the data and to allow actions and meanings to be conceptualised. My memo writing allowed me to immerse myself in the data and the scientist in me to 'experiment' with the emerging themes (Charmaz, 2014, p.162).

I started to build my methodological skills as evidenced in my focus groups and interview transcripts. In the earlier transcripts my voice was dominant, I lacked the skill of picking-up on important points raised and initially found that I had to return and 
question on these later. Importantly, my methodological approach allowed me to do this. I soon identified that a CGT approach was far from the linear process I was used to. This is where CGT methodology really did become my lifejacket and allowed me to negotiate what I saw as the muddy waters of interpretative analysis. The process of concurrent data collection and analysis enabled me to go back and ask more questions, to direct my questioning and develop my analytical direction as I progressed on my journey. I was able to identify 'gaps' and to gather more data to fill these 'gaps' or answer additional questions raised. Had I not started data analysis until all data gathering was complete my findings would not have provided a complete portrayal of the current situation. As my confidence and skills developed I explored perceptions in more depth, allowing participants' voices to dominate and so gather richer and more illuminating data. From a personal perspective, I did not have to be an 'expert' in these from the start. If I had been required to complete data gathering before starting on the analysis stage, I would have fallen at the first hurdle.

\section{Completing my initial journey - completing my doctorate}

My choice of methodology helped me to negotiate and cross boundaries and join the community of educational researchers. It provided a framework, enabling me to learn new skills and approaches that helped me to move from the periphery of this new community of practice and negotiate a space for myself. An important part of my journey was learning that I did not need to change from being a practitioner to being a researcher but rather acquire a new professional identity that encompassed and moved beyond both into 'being in the world' (Wenger, 1998 p.151). However, of equal importance, my methodological approach also enabled me to develop a substantive theory around the role of professional learning and practitioner capability and to successfully complete my doctorate. 


\section{Positioning and Critical Reflexivity}

My doctoral journey helped me to identify that as a scientist and a practitioner, I do not need to reject a positivist or objective epistemology of practice as I evolve in my professional role. I now acknowledge the wider picture, embracing the socio-cultural aspects of learning and the role of questioning and interpretation to develop capability. Importantly, I appreciate that it is not a mutually exclusive concept; I can be a scientist 'who does educational research too'. Recognising this has allowed me to understand the positioning of stakeholders in my enquiry and has supported me to make recommendations for future professional practice. There is a need for practitioners to be encouraged and supported to embrace an appropriate pedagogical culture and develop an appreciation of its role within their own positioning. Based upon my own journey during my professional doctorate I would recommend individuals must acknowledge but more importantly, embrace the barriers resulting from crossing professional boundaries.

\section{Reflections}

A professional doctorate creates opportunities for shared research and novel findings to inform and shape the workplace and cultivate professional capital. As outlined, an epistemological challenge is created when an individual's research spans a diversity of professional domains and multi-professional spaces. Support for those on professional doctorate programmes must acknowledge the distinctiveness of their journey and acknowledge the unique skills, knowledge and experience that they bring with them from their professional and work-based communities. The methodological approach adopted must allow them to embrace and embed their prior experiences rather than reject or put them to one side. In crossing boundaries we must be allowed to bring with us and celebrate the skills and knowledge that we have developed as practitioners 
and to draw upon these skills to inform and shape the workplace and cultivate professional capital. Such an approach will foster the development of novel and original approaches that draw upon and bring together experiences, skills and knowledge from a diversity of professional domains.

\section{References}

Akkerman, S. F. and Bakker, A. 2011. Boundary Crossing and Boundary Objects. Review of Educational Research, 81(2), 132-169.

Birks, M. and Mills, J. 2011. Grounded Theory: A Practical Guide. London: Sage Publications Ltd.

Bryant, A. and Charmaz, K. 2012. Grounded Theory in Historical Perspective: An epistemological Account. in: Bryant, A. and Charmaz, K. (eds) The Sage Handbook of Grounded Theory. London: Sage Publications Ltd. pp.31-57

Burnard, P., Dragovic, T., Ottewell, K. and Lim, W.M., 2018. Voicing the professional doctorate and the researching professional's identity: Theorizing the EdD's uniqueness. London Review of Education, 16(1), 40-55.

Charmaz, K. 2005. Grounded Theory in the 21st Century: Applications for Advancing Social Justice Studies. In Denzin, N. and Lincoln, Y. (eds) The Sage Handbook of Qualitative Research, 3rd Ed, London: Sage Publications Ltd. 507-536 
Charmaz. K. 2014. Constructing Grounded Theory: A Practical Guide Through

Qualitative Analysis (2nd Ed) London: Sage Publications Ltd.

Clarke, A E. 2005. Situational Analysis: Grounded Theory after the Postmodern Turn. Thousand Oaks, Ca: Sage

Goulding, C. 2002 Grounded Theory: A practical guide for management, business and market researchers London: Sage

Holstein, J. and Gubrium, J. 2005. Interpretive Practice and Social Action. In Denzin, N. and Lincoln, Y. (eds) The Sage Handbook of Qualitative Research, 3rd Ed, London: Sage Publications Ltd. 483-506

Holton, J. 2012. The Coding Process and Its Challenges. In: Bryant, A. and Charmaz. K. (eds) The Sage Handbook of Grounded Theory. London: Sage Publications Ltd. 265-290

Opoku, A., Ahmed, V. and Akotia, J., 2016. Choosing an appropriate research methodology and method. In: Ahmed, V., Opoku, A. and Aziz, Z. (eds) Research Methodology in the Built Environment: A selection of case studies. London: Routledge. $52-70$

Smith, S., 2018. Integrated work-based placements-shifting the paradigm. Higher Education, Skills and Work-Based Learning, 8(2), 134-150. 
Star, S.L. and Griesemer, J.R., 1989. Institutional ecology, translations' and boundary objects: Amateurs and professionals in Berkeley's Museum of Vertebrate Zoology, 1907-39. Social studies of science, 19(3), 387-420

Starks, H. and Trinidad, S. B. 2007, 'Choose Your Method: A Comparison of Phenomenology, Discourse Analysis, and Grounded Theory', Qualitative Health Research, 17(10), 1372-1380

Usher, R. (2002). A diversity of doctorates: fitness for the knowledge economy. Higher Education Research \& Development, 21(2), 143-153.

Wenger, E., 1998. Communities of practice: learning, meaning and identity. Cambridge: Cambridge University Press 